\title{
Quantitative Changes in Tumor-Associated M2 Macrophages Characterize Cholangiocarcinoma and their Association with Metastasis
}

\author{
Malinee Thanee ${ }^{1,5}$, Watcharin Loilome ${ }^{1,5 *}$, Anchalee Techasen ${ }^{2,5}$, Nisana \\ Namwat $^{1,5}$, Thidarut Boonmars ${ }^{3,5}$, Chawalit Pairojkul ${ }^{4,5}$, Puangrat Yongvanit ${ }^{1,5 *}$
}

\begin{abstract}
The tumor microenvironment (TME) includes numerous non-neoplastic cells such as leukocytes and fibroblasts that surround the neoplasm and influence its growth. Tumor-associated macrophages (TAMs) and cancerassociated fibroblasts (CAFs) are documented as key players in facilitating cancer appearance and progression. Alteration of the macrophage $(\mathrm{CD68}, \mathrm{CD163})$ and fibroblast $(\alpha-\mathrm{SMA}, \mathrm{FSP}-1)$ cells in Opisthorchis viverrini (Ov) -induced cholangiocarcinoma (CCA) was here assessed using liver tissues from an established hamster model and from 43 human cases using immunohistochemistry. We further investigated whether M2-activated TAMs influence CCA cell migration ability by wound healing assay and Western blot analysis. Macrophages and fibroblasts change their phenotypes to M2-TAMs (CD68+, CD163+) and CAFs ( $\alpha$-SMA+, FSP-1+), respectively in the early stages of carcinogenesis. Interestingly, a high density of the M2-TAMs CCA in patients is significantly associated with the presence of extrahepatic metastases $(p=0.021)$. Similarly, CD163+ CCA cells are correlated with metastases $(\mathrm{p}=\mathbf{0 . 0 0 2})$, and they may be representative of an epithelial-to-mesenchymal transition (EMT) with increased metastatic activity. We further showed that M2-TAM conditioned medium can induce CCA cell migration as well as increase $\mathrm{N}$-cadherin expression (mesenchymal marker). The present work revealed that significant TME changes occur at an early stage of Ov-induced carcinogenesis and that M2-TAMs are key factors contributing to CCA metastasis, possibly via EMT processes.
\end{abstract}

Keywords: Tumor-associated macrophages - cancer-associated fibroblasts - cholangiocarcinoma - metastasis

Asian Pac J Cancer Prev, 16 (7), 3043-3050

\section{Introduction}

The tumor microenvironment (TME) is a complex system composed of many cell types as well as non-cellular components (Albini and Sporn, 2007) that coincide with structural support, tissue homeostasis maintenance, and immune surveillance (Shaykhiev and Bals, 2007; Adjei and Blanka, 2015). The crosstalk between cancer cells and TME is involved in chronic inflammation leading to cancer progression and metastasis through numerous proteins such as CXCL-12, epidermal growth factor (EGF), vascular endothelial growth factor (VEGF), and matrix metalloprotease (MMP) (Joyce and Pollard, 2009; Qian and Pollard, 2010; Rasanen and Vaheri, 2010). These ultimately contribute to unsuccessful wound healing that characterizes cancer(Albini and Sporn, 2007). Changes of stromal cells are reported to occur at the early stages of breast cancer (Weber et al., 2006), and colorectal cancer (Ishiguro et al., 2006) with Smad4-deficient T cells contributing to gastrointestinal cancer onset via the TME, while PTEN inactivation in stromal fibroblasts accelerates breast carcinoma (Trimboli et al., 2009; Adjei and Blanka, 2015).

Tumor-associated macrophages (TAMs) are key players linking inflammation and cancer by promoting tumor cell proliferation and angiogenesis, persistenting matrix turnover, and suppressing adaptive immunity (Solinas et al., 2009). In addition, TAMs contribute many processes in tumor metastasis as well as epithelialmesenchymal transition (EMT) by producing various cytokines (Condeelis and Pollard, 2006; Wang et al., 2014). Among TAMs, M1 macrophages (classically activated cells) are activated by IFN- $\gamma$ and microbial stimuli, such as LPS, and are characterized by high levels of IL-12, IL-23(Martinez et al., 2009), reactive oxygen intermediate (ROI) and possess good capacity as antigen-presenting cells (APC) (Martinez et al., 2009). Conversely, M2 cells are induced by IL-4 and IL-13 (M2a), immune complexes/TLR ligands (M2b), or IL-10 and glucocorticoids (M2c) (Mantovani et al., 2004), which can be defined by several features including high levels of IL-10, IL-1ra, IL-1decoyR and wound-healing promotion

${ }^{1}$ Department of Biochemistry, ${ }^{3}$ Department of Parasitology, ${ }^{4}$ Department of Pathology, ${ }^{5}$ Liver Fluke and Cholangiocarcinoma Research Center, Faculty of Medicine, ${ }^{2}$ Center for Research and Development of Medical Diagnostic Laboratories, Faculty of Associated Medical Sciences, Khon Kaen University, Khon Kaen, Thailand*For correspondence: watloi@yahoo.com; puangrat@kku.ac.th 
(Solinas et al., 2009). A recent report demonstrated that cancer cells and the co-cultured with M2-activated TAMs decrease the expression of epithelial markers and increase the expression of mesenchymal markers in pancreatic and hepatocellular carcinoma (Liu et al., 2013; Wang et al., 2014). This result suggested that M2-activated TAMs is involved in the EMT-related cancer progression. In addition to TAMs, cancer-associated fibroblasts (CAFs) are highly heterogeneous due to their different origins (McAnulty, 2007) with alpha-smooth muscle actin ( $\alpha$-SMA) (Sugimoto et al., 2006), fibroblast specific protein 1 (FSP-1) and platelet-derived growth factor receptor (PDGFR) (Kalluri and Zeisberg, 2006) are used as CAF markers. CAF functions include the secretion of plasminogen activators, MMPs, extracellular matrix (ECM) components, growth factors, and cytokines (De Wever et al., 2008) associated with tumor growth.

The chronic inflammation induced by Opisthorchis viverrini $(\mathrm{Ov})$ infection is the major risk factor for cholangiocarcinoma (CCA) in northeast Thailand (IARC, 1994; Sripa et al., 2007; Sithithaworn et al., 2014; Yongvanit et al., 2014) and is recapitulated in a well-established hamster animal model (Thamavit et al., 1978). In this model, acute inflammatory reaction evolves in hyperplasia and adenomatous lesions of the bile ducts with granulomatous responses and periductal fibrosis(Bhamarapravati et al., 1978). Macrophages and other cells are activated by $\mathrm{Ov}$-specific $\mathrm{T}$ cells and by cytokines as well as nitric oxide (NO) derived from L-arginine via inducible nitric oxide synthase (iNOS) (Yongvanit et al., 2012). Ultimately, Ov-induced fibrogenesis through chronic inflammation (Prakobwong et al., 2009) and NDMA-caused liver injury (Jezequel et al., 1989) may synergistically promote carcinogenesis.

We report herein the first study of TME changes in human and in hamster Ov-induced CCA and demonstrate the transformation of macrophages and fibroblasts to TAM and CAF phenotypes. Additionally, we investigated whether M2-activated TAMs influence CCA cell migration ability possibly through the EMT which is one mechanism of the metastasis processes.

\section{Materials and Methods}

\section{Animal model}

The animal induction protocol and the archive paraffin embedded of Opisthorchis viverrini $(\mathrm{Ov})$ plus $N$-nitrosodimethylamine (NDMA) induced hamster CCA tissues were as previously described (Loilome et al., 2006). Syrian golden hamsters ranging 6-8 weeks of age were housed under conventional conditions and fed stock diet and water ad libitum. Animals were divided into four groups. The treatment group were fed $50 \mathrm{Ov}$ metacercariae by intragastric intubation combined with oral administration of 12.5 ppm NDMA (Sigma, St. Louis, MO) for 8 weeks (Trimboli et al., 2009) while the controls were untreated. The protocol was approved by the Animal Ethics Committee of the Faculty of Medicine, Khon Kaen University (AE002/2002), Thailand. Hamster subgroups were sacrificed and liver tissues collected for paraffin blocks preparation at 7, 14, 21, 30, 90, and 180 days.

\section{Human CCA samples}

Paraffin-embedded liver tissue sections of CCA cases were obtained from the specimen bank of the Liver Fluke and Cholangiocarcinoma Research Center, Faculty of Medicine, Khon Kaen University. Clinical data were available from all subjects and are shown in Table 1. Of the 43 CCA cases, 27 (63\%) were men and the mean age was $58.1 \pm 8.5$ years (range, 37-74 years). Twenty of the 43 patients $(47 \%)$ were at an advanced stage as represented by the presence of extrahepatic metastases. CCA histological types were classified as papillary in $4(9 \%)$ cases and nonpapillary in $39(91 \%)$ cases, respectively. The research protocols were approved by the Human Research Ethics Committee, Khon Kaen University (\#HE521209) and informed consent for the use of specimens was obtained from each subject before surgery.

\section{Cell culture and chemicals.}

Human M214 CCA and macrophage U937 cell lines were cultured in Ham's F12 medium and RPMI medium, respectively supplemented with $\mathrm{NaHCO} 3,100$ units/ml penicillin, $100 \mathrm{mg} / \mathrm{ml}$ streptomysin and $10 \%$ fetal bovine serum. Cells were maintained in a humidified incubator at $37^{\circ} \mathrm{C}$ containing $5 \% \mathrm{CO} 2$. The recombinant human interleukin (IL)-4 was purchased from PeproTech (Israel).

\section{Immunohistochemistry}

Immunohistochemistry was performed for CD68, CD163, $\alpha$-SMA and FSP1 according to standard methods (Hsu and Raine, 1981). Sections of liver tissues were deparaffinized in xylene and rehydrated in a series of concentrations of ethanol. Tissue sections were then irritated for antigen retrieval in $0.05 \%$ Tween20 in 0.1 $\mathrm{M}$ citrate buffer in a microwave oven and placed with $0.3 \% \mathrm{H}_{2} \mathrm{O}_{2}$ for 30 min to block endogenous peroxidase activity. Nonspecific binding was blocked by $10 \%$ skim milk in PBS for $30 \mathrm{~min}$. Sections were incubated overnight with primary antibody at $4^{\circ} \mathrm{C}$ in a moisture chamber. Sections were then incubated with peroxidase-conjugated EnvisionTM secondary antibody (DAKO, Denmark). After washing in working PBS for $5 \mathrm{~min}$, the color was developed with $0.1 \%$ diaminobenzidinetetrahydrochloride solution for 10 min and then counterstained with Mayer's hematoxylin. Sections were observed under a light microscope (Carl Zeiss Axio Scope. A1 microscope) at high magnification power (x400). Macrophage density identified by CD68 expression and M2 macrophage density identified by CD163 expression were estimated (per $\mathrm{mm} 2)$ at high power $(\mathrm{x} 400)$ magnification from 5 areas per case (Ma et al., 2010). The average number of cells in each case was determined as follows: <mean as low density and $>$ mean as high density. CAFs were evaluated by FSP 1 and $\alpha$-SMA staining and the percentage of positive-staining cells was arbitrarily determined as follows: $<5 \%$ positive-staining cells as grade $0,5-25 \%$ positive-staining cells as grade $1,26-50 \%$ positivestaining cells as grade 2 and $>50 \%$ positive-staining cells as grade 3 .

The intensity of staining was graded as grade 0 to grade 2 . For each marker, percentage and intensity scores were multiplied. For FSP1; the final score between 0-1 was 
determined as negative, 2-6 was determined as positive and $\alpha$-SMA; the final score between $0-1$ was determined as negative, 2-3 was determined as low expression, 4-6 was determined as high expression (Zhi et al., 2010). Similar to human CCA patient tissues, in hamster the numbers of macrophages identified by CD68 expression and the numbers of M2 macrophages identified by CD163 expression were estimated at high power (x400) magnification from 5 areas per cases. The average number of macrophage was plotted in graph.

\section{M2-activated TAMs induction}

For M2-activated TAMs, U937 macrophage cells were stimulated with $20 \mathrm{ng} / \mathrm{ml} \mathrm{IL}-4$ for $24 \mathrm{~h}$. After treatment, the cells were changed to be cultured with serum-free RPMI for $48 \mathrm{~h}$. U937 macrophage cells were collected and used as M2-activated TAMs, and the medium was collected for further experimentation.

\section{Cell migration ability using a wound healing assay}

After CCA cells reached confluence, wounds were initiated by scratching by a sterile plastic micropipette tip. After that, CCA cells were treated with condition medium of M2-activated TAMs. The migration of cells into the wound area was observed and photographed by a Nikon Eclipse TS100 microscope.

\section{Western blot analysis}

For protein analysis, CCA cells and M2-activated TAMs were homogenized with lysis buffer and centrifuged at $14000 \mathrm{rpm}$ for $20 \mathrm{~min}$ at $4^{\circ} \mathrm{C}$, and the supernatant was collected.Protein concentration was determined with a BCA kit (Pierce Biotechnology, Rockford, IL). Equal amounts of protein $(30 \mu \mathrm{g})$ were treated with SDS sample buffer and boiled at $95^{\circ} \mathrm{C}$ for $5 \mathrm{~min}$. The samples were separated on $10 \%$ polyacrylamide gels by electrophoresis according to their molecular weight. Proteins were transferred onto polyvinylidenedifluoride membranes. After blocking with $5 \%$ skimmed milk, membrane was incubated with primary antibody overnight at $4^{\circ} \mathrm{C}$ against CD163 and $\mathrm{N}$-cadherin. Subsequently, membrane was incubated with the horseradish peroxidase-conjugated secondary antibody for $1 \mathrm{~h}$ at room temperature. The proteins were then analyzed by enhanced chemiluminescence using the ECL Prime Western Blotting Detection System (GE Healthcare, UK). $\beta$-actin expression was used as an internal control.

\section{Statistical analysis}

Statistical comparisons were performed using SPSS software version 17.0 (IBM Corporation, Armonk, NY). The association between CD68, CD163, $\alpha$-SMA and FSP1 expression in CCA tissue and patient clinical and pathological data were assessed by chi- square test. The patient-survival was calculated according to KaplanMeier, with a log-rank test. All analyses were two-tailed and P-values $<0.05$ were considered as statistically significant.

\section{Results}

Macrophage and fibroblast phenotypes in cholangiocarcinogenesis

As expected, CCA fully developed at 180 days in Ov plus NDMA-treated hamsters while no histopathological

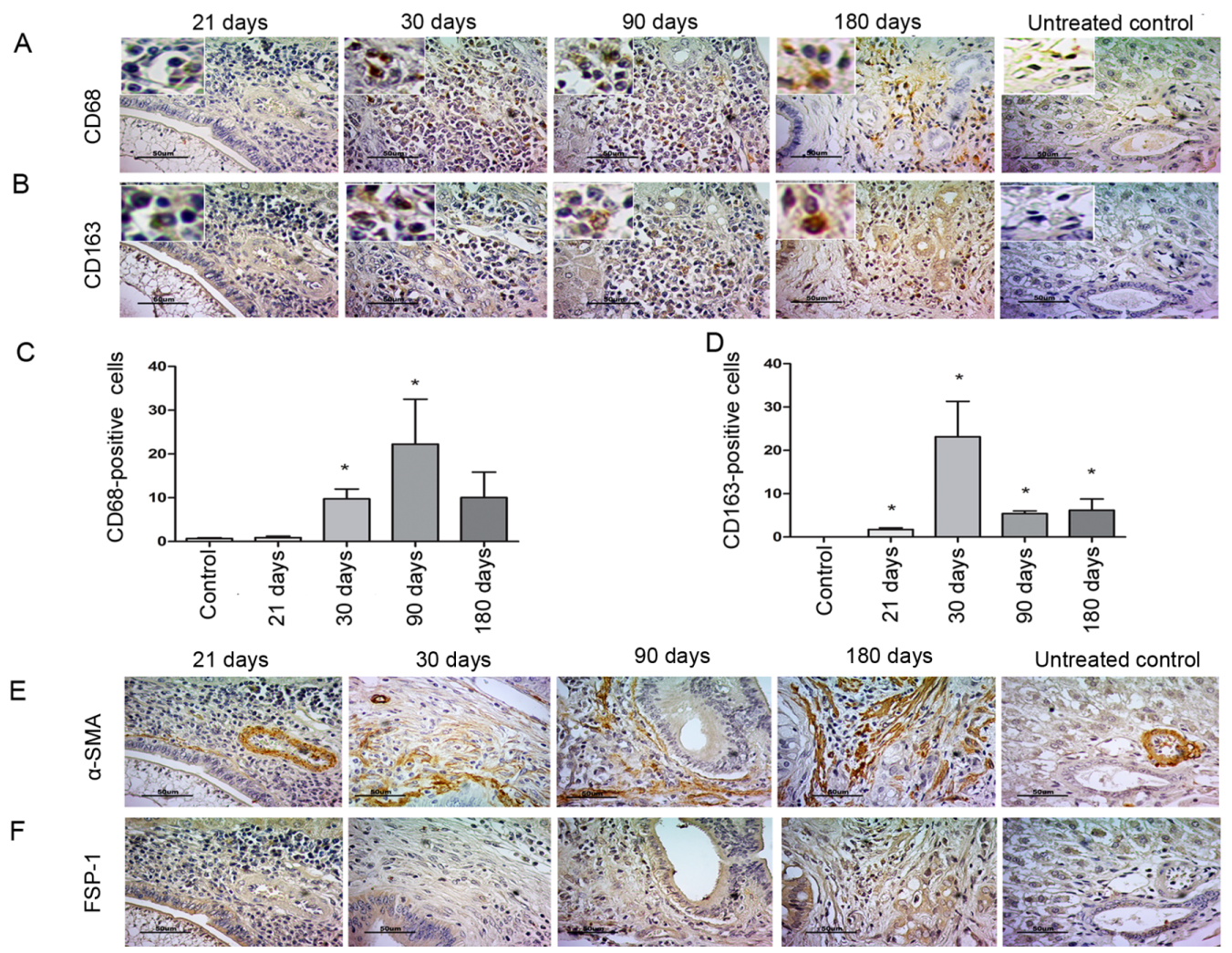

Figure 1. Immunohistochemistry Staining Results. CAFs markers in liver tissues of Ov-infected hamsters with NDMA administration and untreated control. (A) CD68 macrophage marker; (B) CD163 M2-macrophage marker; (C, D) average number of CD68- and CD163-positive cells in hamster liver tissues; (E) $\alpha$-SMA and (F) FSP-1. Magnification 40x; Ov, Opisthorchis viverrini. 
Table 1. Clinical Features of Patients with CCA Enrolled in the Study. Continuous Variables are Expressed as Mean \pm Standard Deviation (Range)

\begin{tabular}{lc}
\hline Patients (n) & 43 \\
\hline Age (years) & $58 \pm 9(37-74)$ \\
Male (n) & $27 / 43(63 \%)$ \\
Histology (n) & $39(91 \%)$ \\
Non-papillary type & $4(9 \%)$ \\
Papillary type & $427 \pm 82(3-2200)$ \\
Survival Day (days) & $20 / 43(46 \%)$ \\
Patients with metastases (n) & \\
\hline
\end{tabular}

change in bile duct epithelial cells was observed in the control hamsters. In the former group, the transformation of macrophages to TAMs and fibroblasts to CAFs occurs at the early CCA stage. CD68 (macrophage marker) and CD163 (M2-macrophage marker) staining was slight in liver tissues obtained from control hamsters. A few positive signals of CD68 and CD163 were detected at days 7,14 (data not shown) and 21. Marked expressions of both markers were observed at day 30 while CD68 showed the highest positive expression at day 90 (Figure 1A-B). In addition, TAM positive signal intensity increased from hyperplasia at day 30, to dysplasia at day 90, and CCA at day 180 where the strongest signal was found (Figure 1A-B). The presence of CAFs in liver tissues of Ov plus NDMA-treated hamsters was observed. The $\alpha$-SMA positive CAFs were associated with CCA development in parallel with FSP1 (Figure 1E-F). Moreover, FSP-1 expression was also found in transformed bile duct cells from hyperplasia at day 30 , to dysplasia at day 90 , and cancer at day 180 (Figure 1F).

\section{TAMs and CAFs in human CCA}

CD68 and CD163 positive inflammatory cells were detected in human CCA liver tissues and adjacent areas with similar intensity (Figure 2A). High CD68 and CD163 expression was cumulative in 53\% (23/43) and 51\% (22/43) of CCA cases (Table 2). Moreover, expressions of CD68 and CD163 in the cancer bile ducts were found in $12 \%(5 / 43)$ and $72 \%(31 / 43)$ of CCA cases (Figure 2B, Table 2). We also observed a high expression of

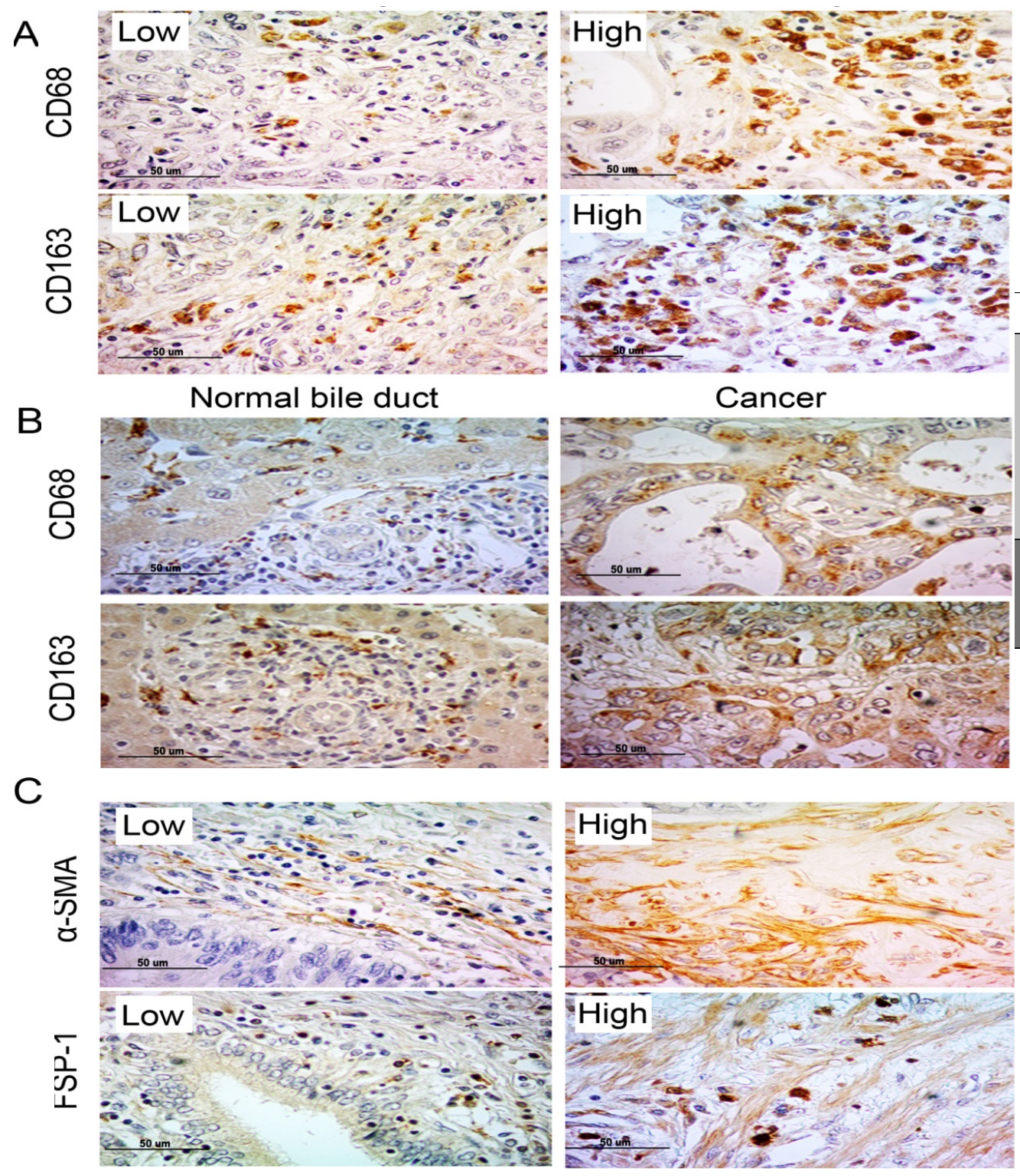

Figure 2. Staining Results. (A) Low and High density of CD68- and CD163-positive inflammatory cells in CCA. (B) Expression of CD68 and CD163 in the cancer cells were determined by immunohistochemical staining. (C) Low and high expression of $\alpha$-SMA and FSP-1 in stromal cells of CCA liver tissues. Magnification 40x 


\begin{tabular}{|c|c|c|c|c|c|c|c|c|c|c|}
\hline \multirow[t]{3}{*}{$\begin{array}{l}\text { Clinicopathologic } \\
\text { data }\end{array}$} & \multirow[t]{3}{*}{$\mathrm{N}$} & \multicolumn{6}{|c|}{$\begin{array}{l}\text { Density of TAM } \\
\text { in liver tissues }\end{array}$} & \multicolumn{3}{|c|}{$\begin{array}{l}\text { Expression of CD163 } \\
\text { in CCA cells }\end{array}$} \\
\hline & & \multicolumn{2}{|c|}{ CD68 } & \multirow[b]{2}{*}{$\mathrm{P}$} & \multicolumn{2}{|c|}{ CD163 } & \multirow[b]{2}{*}{$\mathrm{P}$} & \multirow{2}{*}{\multicolumn{2}{|c|}{$\frac{\mathrm{CD} 163}{\text { negative positive }}$}} & \multirow[b]{2}{*}{$\mathrm{P}$} \\
\hline & & high & low & & high & low & & & & \\
\hline \multicolumn{11}{|l|}{ Age (years) } \\
\hline$<58$ & 24 & 15 & 9 & 0.228 & 13 & 11 & 0.763 & 8 & 16 & 0.5 \\
\hline$\geq 58$ & 19 & 8 & 11 & & 9 & 10 & & 4 & 15 & \\
\hline \multicolumn{11}{|l|}{ Sex } \\
\hline Male & 27 & 12 & 15 & 0.122 & 16 & 11 & 0.168 & 8 & 19 & 1 \\
\hline Female & 16 & 11 & 5 & & 6 & 10 & & 4 & 12 & \\
\hline \multicolumn{11}{|l|}{ Histological types } \\
\hline non papillary & 39 & 21 & 18 & 1 & 20 & 19 & 1 & 11 & 28 & 1 \\
\hline papillary & 4 & 2 & 2 & & 2 & 2 & & 1 & 3 & \\
\hline \multicolumn{11}{|l|}{ Metastasis } \\
\hline no metastasis & 23 & 14 & 9 & 0.298 & 8 & 15 & $0.021 *$ & 11 & 12 & $0.002 *$ \\
\hline metastasis & 20 & 9 & 11 & & 14 & 6 & & 1 & 19 & \\
\hline
\end{tabular}

*P value equal to or less than 0.05 was considered statistically significant

$\alpha$-SMA and FSP-1 in stromal cells surrounding the tumor area in $83.7 \%(36 / 43), 27.9 \%(12 / 43)$ of CCA patients respectively (Figure $2 \mathrm{C}$ ).

\section{TAMs and CAFs expression and their clinical correlations}

Our results utilizing mean cell numbers to identify clinical subgroups show that patients with higher density of CD163 positive cells have significantly more frequent extrahepatic metastasis (Table 2). We further observed that patients with a high density of CD68 positive cells had longer survival. Conversely, a higher density of CD163 positive cells in liver tissues was associated with shorter survival, albeit that difference was not statistically significant (data not shown). Interestingly, a higher density of CD163 positive inflammatory cells in liver tissues was also associated tumor metastasis (Table 2).

No correlation between $\alpha$-SMA and FSP-1 expression and clinical-pathological features was observed (data not shown).

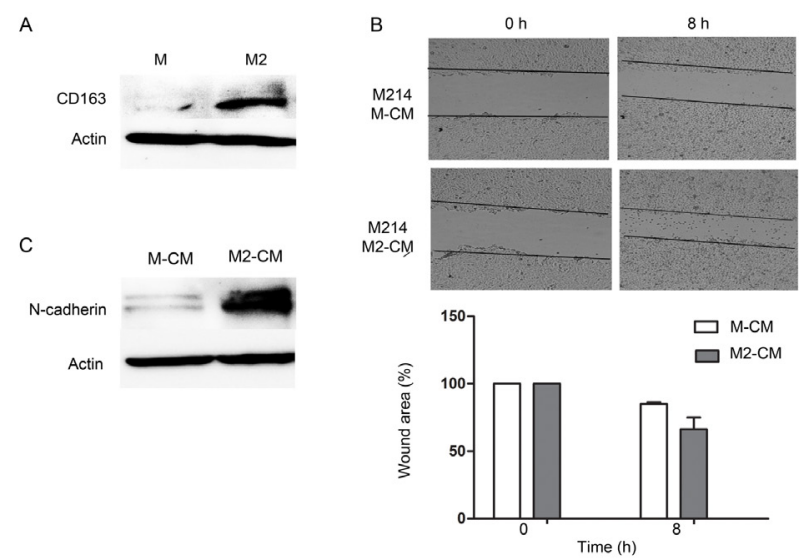

Figure 3. Results. (A) Western blotting shows expression of CD163 (M2 marker) in M: U937 macrophage cell and M2: M2activated TAM via IL-4 stimulation. (B) Wound healing assays showing M214 CCA cells stimulated by M2-activated TAMs conditioned medium (M214 M2-CM) and M214 in macrophage control conditioned medium (M214 M-CM) at 0 and $8 \mathrm{~h}$. The percent of wound area was recorded in each group. (C) The expression of $\mathrm{N}$-cadherin in M214 CCA cells stimulated by M2-activated TAMs conditioned medium (M2-CM) and M214 in macrophage control conditioned medium (M-CM)
Effect of M2-activated TAMs conditioned medium on CCA cells migration ability

Our results show that high CD163 positive staining in both cancer cells and surrounding inflammatory cells was significantly associated with the metastasis status of patients. We investigated whether this phenomenon was due to, at least in part, the EMT process. After IL-4 treatment, the CD163, scavenger receptor was significantly higher in activated macrophages than native macrophages (Figure 3A). This result suggested that 20 $\mathrm{ng} / \mathrm{ml}$ of IL-4 successfully induced the alteration of M2activated TAMs. We examined the effect of M2-activated TAMs on CCA cell migration by the wound healing assay. As shown in Figure 3B, CCA cells cultured with M2-activated TAMs-conditioned medium (M2-CM) had the scratch slightly narrower than CCA cells cultured with untreated macrophage-conditioned media (M-CM). Additionally, the level of the $\mathrm{N}$-cadherin, mesenchymal marker was observed to increase in this condition (Figure $3 \mathrm{C})$. These results suggested that M2-macrophage may secrete some cytokines to enhance the migration behavior of CCA cells.

\section{Discussion}

Current treatment strategies for CCA are largely ineffective. For this reason, it is of great importance to identify new pathogenetic mechanisms for potential therapeutic implications. The present study takes advantage of an established Ov-infected animal model and a large series of defined CCA cases to demonstrate for the first time that TME is significantly altered and that this is largely dependent on TAMs and CAFs. In particular, we observed that altered macrophages are M2-TAMs which are found at early stages of cholangiocarcinogenesis. Most importantly, we could discriminate a clinical correlation with a more aggressive and advanced disease as represented by extrahepatic metastases in human CCA.

TAMs are well known to play a role in carcinogenesis and metastasis (Solinas et al., 2009; Qian and Pollard, 2010) and it is widely accepted that CD68 and CD163 identify M2-TAMs in breast cancer (Medrek et al., 
2012), and CCA (Hasita et al., 2010). The present study confirms data from our group supporting that $\mathrm{Ov}$ infection induces an inflammatory response through a TLR2-mediated pathway leading to nuclear factorkappa beta (NF- $\kappa \beta)$-mediated expression of iNOS and cyclooxygenase (COX)-2 in macrophage cell line (Pinlaor et al., 2005). Furthermore, in vivo evidence demonstrated that inflammatory macrophage-like cells expressions and MMP-9 that may concur to DNA damage and CCA development (Prakobwong et al., 2010). We also confirmed that $\mathrm{Ov}$ infection induces a TME alteration associated with macrophage transformation to M2-TAMs which may produce reactive nitrogen intermediates (RNI) and MMP-9, possibly pivotal to CCA onset. Moreover, M2-TAMs might also be associated with a high progression of cancer, since MMP-9 expression has been observed in inflammatory cells (Prakobwong et al., 2010). Of particular importance is that pre-neoplastic lesions, such as intestinal adenomas and chronic obstructive pulmonary disease (COPD), also share an increased inflammatory milieu including macrophages. This has important implications for colorectal (McLean et al., 2011)and lung carcinogenesis (Moghaddam et al., 2009).

A high density of M2-phenotype TAMs (characterized by CD163) was significantly correlated with extrahepatic metastases, consistent with reports of the association between CD14+CD16+ monocytes and rapid tumor progression (Subimerb et al., 2010b). Moreover, high expression of the MAC387 macrophage marker is associated with worst case prognosis of CCA patient (Subimerb et al., 2010a). Hasitaet and co-workers reported that TAMs contribute to cancer progression via STAT3 activation and that TAMs quantitatively correlated with poor prognosis in breast (Tsutsui et al., 2005), colon (Forssell et al., 2007), thyroid (Kim et al., 2013) and oral squamous (Ni et al., 2015) cancer. Similarly, TAMs also correlate with metastatic disease in colorectal (Kang et al., 2010), lung (Zhang et al., 2011) pancreatic (Chen et al., 2015) cancer and liposarcoma (Nabeshima et al., 2015) while they appear to be protective in gastric carcinoma (Ohno et al., 2003). The current views imply different mechanisms of action for TAMs including activated macrophages in inflammation, immune regulation by immunosuppressive M1 macrophages, promoting angiogenesis by secreting angiogenic factor, perivascular macrophage contributing to tumor invasion, and metastasis (Qian and Pollard, 2010).

Similar to TAMs, CAFs may also promote carcinogenesis and metastasis via different mechanisms (Rasanen and Vaheri, 2010) and it is therefore of great importance that fibroblasts are altered at early stages of Ov-associated bile duct cell transformation to CCA, as previously suggested (Prakobwong et al., 2010). CAFs support tumorigenesis by stimulating angiogenesis, cancer cell proliferation and invasion, which are well demonstrated by macrophage recruitment, neovascularization, and tumor growth in the hyperplastic stage in skin tumorigenesis (Erez et al., 2010) and data support a direct role in metastases from gastric (Zhi et al., 2010), and breast (Huang et al., 2010) cancer. In the present study we found no significant correlation between CAFs and the patients' clinical features but we did note that CCA cases with higher $\alpha$-SMA and FSP-1 expression tend to have shorter survival time, once again confirming previous observations on $\alpha$-SMA expressing fibroblasts (Chuaysri et al., 2009). The same authors also reported that $\alpha$-SMA positive fibroblasts induce the proliferation of non-neoplastic biliary epithelial and CCA cells in early stages of carcinogenesis, with fibroblasts inducing uncontrolled growth by apoptosis inhibition. In later disease stages, biliary epithelial cells are completely transformed to CCA and the induction of cell proliferation becomes predominant (Chuaysri et al., 2009). Moreover, current study also suggested that $\alpha$-SMA positive fibroblasts induce cancer growth and migration (Campbell et al., 2012). Additionally, deleting CAF by navitoclax treatment can suppress tumor growth, and improve host survival in rat CCA model (Mertens et al., 2013).

Complementary to our animal data, the cross-sectional study of 43 CCA samples demonstrates that patients who expressed CD163 more frequently had extrahepatic metastatic disease. Neoplastic cells express macrophage markers such as CD68 and CD163 in breast (Shabo et al., 2008) and bladder (Maniecki et al., 2012) cancer, and the proportion of CD163-expressing tumor cells correlates with metastasis and shorter survival. These observations have led to the fascinating hypothesis of a fusion between cancer cells and TAMs forming hybrids that are highly likely to set distant metastases (Shabo et al., 2008). Further, CD163-expressing cancer cells may manifest an EMT-associated phenotypic shift and increased metastatic activity induced by TAMs, a paradigm of the cellular interaction between cancer and immunity (Maniecki et al., 2012). Nevertheless, FSP-1 is expressed in CCA cells despite the absence of significant clinical-pathological correlations and this follows previous reports that FSP-1 is expressed in metastatic cancer (Ebralidze et al., 1989) and in colorectal carcinomas (Sack et al., 2011). One consequence of this observation is that FSP-1 may trigger EMT activation.

FSP-1 is commonly used as a marker to identify epithelial cells undergoing EMT during tissue fibrogenesis (Iwano et al., 2002)in hepatocytes (Zeisberg et al., 2007) and cholangiocytes (Diaz et al., 2008). The expression of FSP-1 also by inflammatory cells may represent a specific subset of inflammatory macrophages involved in liver injury, fibrosis, and cancer (Osterreicher et al., 2011). We propose that a highly invasive CCA phenotype may express TAM and/or CAF markers. Additionally, we investigated whether M2-activated TAMs influence CCA cell migration ability. Our results showed that IL4stimulated M2 macrophages conditioned medium can induce CCA cells migration as demonstrated by a wound healing assay. $\mathrm{N}$-cadherin is an effective mesenchymal marker for checking alteration of cancer metastatic ability (Hazan et al., 2000; Techasen et al., 2014). In our model, the level of $\mathrm{N}$-cadherin increased with M2-activated TAMs stimulated CCA cells. The present work revealed that significant TME changes occur at an early stage of Ovinduced carcinogenesis and that the accumulation of M2TAMs may be linked to the presence of CCA metastasis.

In conclusion, our data identify changes in the 
microenvironment of pre-CCA and invasive CCA liver tissues as well as point to macrophages and fibroblasts as major mediators of these phenomena. We have shown that a phenotypic change of macrophages and fibroblasts occurs early in Ov-associated CCA and is accompanied by the expression of M2-TAM and CAF markers. These data are consistent with similar observations in human CCA tissues. Interestingly, a high accumulation of M2-TAM in CCA was related to metastasis potency which possibly mediated by EMT processes. The data cumulatively suggest that M2-TAMs and CAFs may represent potential targets for CCA prevention and treatment.

\section{Acknowledgements}

This research was supported by Thailand Research Fund through Royal Golden Jubilee Ph.D. Program (to MT and PY), Khon Kaen University Research Fund, and Grant from Faculty of Medicine (Grant No. I55203), Khon Kaen University in Thailand. This work was supported by the Higher Education Research Promotion and National Research University Project of Thailand, Office of the Higher Education Commission, through the Center of Excellence in Specific Health Problems in Greater Mekong Sub-region cluster (SHeP-GMS), Khon Kaen University to WL. We thank Prof. Ross H. Andrews for English assistance.

\section{References}

Adjei IM, Blanka S (2015). Modulation of the tumor microenvironment for cancer treatment: a biomaterials approach. J Funct Biomater, 6, 81-103.

Albini A, Sporn MB (2007). The tumour microenvironment as a target for chemoprevention. Nat Rev Cancer, 7, 139-47.

Bhamarapravati N, Thammavit W, Vajrasthira S (1978). Liver changes in hamsters infected with a liver fluke of man, Opisthorchis viverrini. Am J Trop Med Hyg, 27, 787-94.

Campbell DJ, Dumur CI, Lamour NF, et al (2012). Novel organotypic culture model of cholangiocarcinoma progression. Hepatol Res, 42, 1119-30.

Chen SJ, Zhang QB, Zeng LJ, et al (2015). Distribution and clinical significance of tumour-associated macrophages in pancreatic ductal adenocarcinoma: a retrospective analysis in China. Curr Oncol, 22, 11-9.

Chuaysri C, Thuwajit P, Paupairoj A, et al (2009). Alphasmooth muscle actin-positive fibroblasts promote biliary cell proliferation and correlate with poor survival in cholangiocarcinoma. Oncol Rep, 21, 957-69.

Condeelis J, Pollard JW (2006). Macrophages: obligate partners for tumor cell migration, invasion, and metastasis. Cell, 124, 263-6.

De Wever O, Demetter P, Mareel M, et al (2008). Stromal myofibroblasts are drivers of invasive cancer growth. Int $J$ Cancer, 123, 2229-38.

Diaz R, Kim JW, Hui JJ, et al (2008). Evidence for the epithelial to mesenchymal transition in biliary atresia fibrosis. Hum Pathol, 39, 102-15.

Ebralidze A, Tulchinsky E, Grigorian M, et al (1989). Isolation and characterization of a gene specifically expressed in different metastatic cells and whose deduced gene product has a high degree of homology to a $\mathrm{Ca} 2+$-binding protein family. Genes Dev, 3, 1086-93.

Erez N, Truitt M, Olson P, et al (2010). Cancer-Associated
Fibroblasts Are Activated in Incipient Neoplasia to Orchestrate Tumor-Promoting Inflammation in an NFkappaB-Dependent Manner. Cancer Cell, 17, 135-47.

Forssell J, Oberg A, Henriksson ML, et al (2007). High macrophage infiltration along the tumor front correlates with improved survival in colon cancer. Clin Cancer Res, 13, 1472-9.

Hasita H, Komohara Y, Okabe H, et al (2010). Significance of alternatively activated macrophages in patients with intrahepatic cholangiocarcinoma. Cancer Sci, 101, 1913-9.

Hazan RB, Phillips GR, Qiao RF, et al (2000). Exogenous expression of $\mathrm{N}$-cadherin in breast cancer cells induces cell migration, invasion, and metastasis. J Cell Biol, 148, 779-90.

Hsu SM, Raine L (1981). Protein A, avidin, and biotin in immunohistochemistry. J Histochem Cytochem, 29, 1349-53.

Huang M, Li Y, Zhang H, et al (2010). Breast cancer stromal fibroblasts promote the generation of CD44+CD24- cells through SDF-1/CXCR4 interaction. J Exp Clin Cancer Res, 29, 80.

IARC (1994). Infection with liver flukes (Opisthorchis viverrini, Opisthorchis felineus and Clonorchis sinensis). IARC Monogr Eval Carcinog Risks Hum, 61, 121-75.

Ishiguro K, Yoshida T, Yagishita H, et al (2006). Epithelial and stromal genetic instability contributes to genesis of colorectal adenomas. Gut, 55, 695-702.

Iwano M, Plieth D, Danoff TM, et al (2002). Evidence that fibroblasts derive from epithelium during tissue fibrosis. $J$ Clin Invest, 110, 341-50.

Jezequel AM, Mancini R, Rinaldesi ML, et al (1989). Dimethylnitrosamine-induced cirrhosis. Evidence for an immunological mechanism. J Hepatol, 8, 42-52.

Joyce JA, Pollard JW (2009). Microenvironmental regulation of metastasis. Nat Rev Cancer, 9, 239-52.

Kalluri R, Zeisberg M (2006). Fibroblasts in cancer. Nat Rev Cancer, 6, 392-401.

Kang JC, Chen JS, Lee CH, et al (2010). Intratumoral macrophage counts correlate with tumor progression in colorectal cancer. J Surg Oncol, 102, 242-8.

Kim S, Cho SW, Min HS, et al (2013). The expression of tumorassociated macrophages in papillary thyroid carcinoma. Endocrinol Metab (Seoul), 28, 192-8.

Liu CY, Xu JY, Shi XY, et al (2013). M2-polarized tumorassociated macrophages promoted epithelial-mesenchymal transition in pancreatic cancer cells, partially through TLR4/ IL-10 signaling pathway. Lab Invest, $\mathbf{9 3}, 844-54$.

Loilome W, Yongvanit P, Wongkham C, et al (2006). Altered gene expression in Opisthorchis viverrini-associated cholangiocarcinoma in hamster model. Mol Carcinog, 45, 279-87.

Ma J, Liu L, Che G, et al (2010). The M1 form of tumorassociated macrophages in non-small cell lung cancer is positively associated with survival time. BMC Cancer, 10, 112 .

Maniecki MB, Etzerodt A, Ulhoi BP, et al (2012). Tumorpromoting macrophages induce the expression of the macrophage-specific receptor CD163 in malignant cells. Int J Cancer, 131, 2320-31.

Mantovani A, Sica A, Sozzani S, et al (2004). The chemokine system in diverse forms of macrophage activation and polarization. Trends Immunol, 25, 677-86.

Martinez FO, Helming L, Gordon S (2009). Alternative activation of macrophages: an immunologic functional perspective. Аnпи Rev Immunol, 27, 451-83.

McAnulty RJ (2007). Fibroblasts and myofibroblasts: their source, function and role in disease. Int $J$ Biochem Cell Biol, 39, 666-71.

McLean MH, Murray GI, Stewart KN, et al (2011). The 
inflammatory microenvironment in colorectal neoplasia. PLoS One, 6, 15366.

Medrek C, Ponten F, Jirstrom K, et al (2012). The presence of tumor associated macrophages in tumor stroma as a prognostic marker for breast cancer patients. BMC Cancer, 12, 306

Mertens JC, Fingas CD, Christensen JD, et al (2013). Therapeutic effects of deleting cancer-associated fibroblasts in cholangiocarcinoma. Cancer Res, 73, 897-907.

Moghaddam SJ, Li H, Cho SN, et al (2009). Promotion of lung carcinogenesis by chronic obstructive pulmonary diseaselike airway inflammation in a K-ras-induced mouse model. Am J Respir Cell Mol Biol, 40, 443-53.

Nabeshima A, Matsumoto Y, Fukushi J, et al (2015). Tumourassociated macrophages correlate with poor prognosis in myxoid liposarcoma and promote cell motility and invasion via the HB-EGF-EGFR-PI3K/Akt pathways. Br J Cancer, 112, 547-55.

Ni YH, Ding L, Huang XF, et al (2015). Microlocalization of CD68 tumor-associated macrophages in tumor stroma correlated with poor clinical outcomes in oral squamous cell carcinoma patients. Tumour Biol.

Ohno S, Inagawa H, Dhar DK, et al (2003). The degree of macrophage infiltration into the cancer cell nest is a significant predictor of survival in gastric cancer patients. Anticancer Res, 23, 5015-22.

Osterreicher $\mathrm{CH}$, Penz-Osterreicher M, Grivennikov SI, et al (2011). Fibroblast-specific protein 1 identifies an inflammatory subpopulation of macrophages in the liver. Proc Natl Acad Sci U S A, 108, 308-13.

Pinlaor S, Tada-Oikawa S, Hiraku Y, et al (2005). Opisthorchis viverrini antigen induces the expression of Toll-like receptor 2 in macrophage RAW cell line. Int J Parasitol, 35, 591-6.

Prakobwong S, Pinlaor S, Yongvanit P, et al (2009). Time profiles of the expression of metalloproteinases, tissue inhibitors of metalloproteases, cytokines and collagens in hamsters infected with Opisthorchis viverrini with special reference to peribiliary fibrosis and liver injury. Int J Parasitol, 39, 825-35.

Prakobwong S, Yongvanit P, Hiraku Y, et al (2010). Involvement of MMP-9 in peribiliary fibrosis and cholangiocarcinogenesis via Rac1-dependent DNA damage in a hamster model. Int $J$ Cancer, 127, 2576-87.

Qian BZ, Pollard JW (2010). Macrophage diversity enhances tumor progression and metastasis. Cell, 141, 39-51.

Rasanen K, Vaheri A (2010). Activation of fibroblasts in cancer stroma. Exp Cell Res, 316, 2713-22.

Sack U, Walther W, Scudiero D, et al (2011). S100A4-induced cell motility and metastasis is restricted by the Wnt/betacatenin pathway inhibitor calcimycin in colon cancer cells. Mol Biol Cell, 22, 3344-54.

Shabo I, Stal O, Olsson H, et al (2008). Breast cancer expression of CD163, a macrophage scavenger receptor, is related to early distant recurrence and reduced patient survival. Int $J$ Cancer, 123, 780-6.

Shaykhiev R, Bals R (2007). Interactions between epithelial cells and leukocytes in immunity and tissue homeostasis. $J$ Leukoc Biol, 82, 1-15.

Sithithaworn P, Yongvanit P, Duenngai K, et al (2014). Roles of liver fluke infection as risk factor for cholangiocarcinoma. $J$ Hepatobiliary Pancreat Sci, 21, 301-8.

Solinas G, Germano G, Mantovani A, et al (2009). Tumorassociated macrophages (TAM) as major players of the cancer-related inflammation. J Leukoc Biol, 86, 1065-73.

Sripa B, Kaewkes S, Sithithaworn P, et al (2007). Liver fluke induces cholangiocarcinoma. PLoS Med, 4, 201.

Subimerb C, Pinlaor S, Khuntikeo N, et al (2010a). Tissue invasive macrophage density is correlated with prognosis in cholangiocarcinoma. Mol Med Report, 3, 597-605.

Subimerb C, Pinlaor S, Lulitanond V, et al (2010b). Circulating CD14(+) CD16(+) monocyte levels predict tissue invasive character of cholangiocarcinoma. Clin Exp Immunol, 161, 471-9.

Sugimoto H, Mundel TM, Kieran MW, et al (2006). Identification of fibroblast heterogeneity in the tumor microenvironment. Cancer Biol Ther, 5, 1640-6.

Techasen A, Namwat N, Loilome W, et al (2014). Tumor necrosis factor-alpha modulates epithelial mesenchymal transition mediators ZEB2 and S100A4 to promote cholangiocarcinoma progression. J Hepatobiliary Pancreat Sci, 21, 703-11.

Thamavit W, Bhamarapravati N, Sahaphong S, et al (1978). Effects of dimethylnitrosamine on induction of cholangiocarcinoma in Opisthorchis viverrini-infected Syrian golden hamsters. Cancer Res, 38, 4634-9.

Trimboli AJ, Cantemir-Stone CZ, Li F, et al (2009). Pten in stromal fibroblasts suppresses mammary epithelial tumours. Nature, 461, 1084-91.

Tsutsui S, Yasuda K, Suzuki K, et al (2005). Macrophage infiltration and its prognostic implications in breast cancer: the relationship with VEGF expression and microvessel density. Oncol Rep, 14, 425-31.

Wang X, Wang H, Li G, et al (2014). Activated macrophages down-regulate expression of E-cadherin in hepatocellular carcinoma cells via NF-kappaB/Slug pathway. Tumour Biol, 35, 8893-901.

Weber F, Shen L, Fukino K, et al (2006). Total-genome analysis of BRCA1/2-related invasive carcinomas of the breast identifies tumor stroma as potential landscaper for neoplastic initiation. Am J Hum Genet, 78, 961-72.

Yongvanit P, Pinlaor S, Bartsch H (2012). Oxidative and nitrative DNA damage: key events in opisthorchiasis-induced carcinogenesis. Parasitol Int, 61, 130-5.

Yongvanit P, Pinlaor S, Loilome W (2014). Risk biomarkers for assessment and chemoprevention of liver fluke-associated cholangiocarcinoma. J Hepatobiliary Pancreat Sci, 21, 309-15.

Zeisberg M, Yang C, Martino M, et al (2007). Fibroblasts derive from hepatocytes in liver fibrosis via epithelial to mesenchymal transition. J Biol Chem, 282, 23337-47.

Zhang BC, Gao J, Wang J, et al (2011). Tumor-associated macrophages infiltration is associated with peritumoral lymphangiogenesis and poor prognosis in lung adenocarcinoma. Med Oncol, 28, 1447-52.

Zhi K, Shen X, Zhang H, et al (2010). Cancer-associated fibroblasts are positively correlated with metastatic potential of human gastric cancers. J Exp Clin Cancer Res, 29, 66. 